\title{
Brain metastases from patients with breast cancer coordinately down-regulate a network of collagen genes.
}

\author{
Shahan Mamoor ${ }^{1}$ \\ 1Thomas Jefferson School of Law \\ San Diego, CA 92901 \\ mamoorsk@tjsl.edu
}

Breast cancer is one of the most common cancers in women and a leading cause of death for women (1). Metastasis, the spread of the cancer from the site of the primary tumor to a foreign site, is, in general, the reason why humans die from cancer (2). There are limited treatment options for women with metastatic breast cancer, which can spread from the breast to the brain (3). We compared the transcriptomes of 16 breast tumors to the transcriptomes of 16 brain metastases from the same patients using a published dataset (4). We discovered that 12 independent genes of the collagen family were among the genes whose expression was most different between primary breast tumors in humans and the brain metastases that they generate when considering the entire transcriptome. Each of these collagen genes were expressed at significantly lower levels in metastases to the brain than in tumors of the breast. The biology of collagen genes in the metastatic process in breast cancer should be evaluated as a means to developing novel therapeutic strategies in metastatic breast cancer.

Keywords: breast cancer, brain metastasis, systems oncology, tumor transcriptome analysis, collagen, extracellular matrix. 


\section{Introduction}

Breast cancer is the second leading cause of cancer in women and one of the leading causes of death for women (1). In a single year, there were 63,410 cases of breast cancer in situ, 252,710 cases of invasive breast cancer, and 40,610 deaths from breast cancer (1). $90 \%$ of cancer deaths are caused by metastasis, or spread of the cancer to a foreign site (2). Systems-level analysis of the transcriptomes of breast tissues by global differential gene expression analysis provides the opportunity to identify the most significant differences in the total transcriptional output between any given comparison of cell state or tissue type and illuminate the basic transcriptional behavior of the cancer transcriptome (5).

In this study, we compared the transcriptomes of breast tumors and brain metastases from breast tumors from 16 patients to determine in an unbiased, systematic fashion the most significant changes in the gene expression between primary breast tumors and the brain metastases that they generate using a published dataset. We found that 12 separate genes of the collagen family, genes whose products serve as structural components in the extracellular matrix (6), were among the most differentially expressed genes transcriptome-wide when comparing breast tumors to brain metastases from the same patients. Moreover, each of these genes was expressed at significantly lower levels in brain metastases than in breast tumors. These are biologically significant findings as manipulation of the extracellular matrix is a key process in metastasis $(7)$.

\section{Methods}

GSE125989 (4) was utilized for this analysis in conjunction with GEO2R. Investigators that created GSE125989 used Affymetrix Human Genome U133A 2.0 microarray technology. In this study, the Benjamin and Hochberg method of p-value adjustment was utilized, and logtransformation of data was set to "Auto-detect". The raw p-value is utilized here. The NCBI generated category of platform annotation was utilized. A statistical test was performed to evaluate the significance of difference in RNA expression values between each differentially 
expressed gene in primary breast tumors and brain metastases by a two-tailed, unpaired t-test with Welch's correction using PRISM (8.1.2) (227). Computational protein-protein interaction network analysis was performed using STRING (8) to search for interactions low-confidence interactions in Homo sapiens, but only interactions that were experimentally determined were considered in the query.

\section{Results}

We performed global gene expression profiling of 16 primary tumors isolated from patients diagnosed with breast cancer, and compared their transcriptomes to that of brain metastases isolated from the same patients, using a published microarray dataset. We found twelve collagen genes (right, and Table 1) that were among the genes whose expression changed the most significantly, transcriptome-wide, between breast tumor and brain metastases.

COL1A1, collagen type I alpha 1 chain, was ranked 2 nd out of 22277 total transcripts measured and detected by the microarray dataset utilized in this study. This was statistically significant (Table 1; $\mathrm{p}=1.02 \mathrm{E}-08)$.

COL15A1, collagen type XV alpha 1 chain, was ranked 6th out of 22277 total transcripts measured and detected by the microarray dataset

\begin{tabular}{|c|c|c|}
\hline$\underline{\text { Rank }}$ & Gene & Gene Name \\
\hline 2 & COL1A1 & $\begin{array}{l}\text { collagen type I } \\
\text { alpha } 1 \text { chain }\end{array}$ \\
\hline 6 & COL15A1 & $\begin{array}{l}\text { collagen type XV } \\
\text { alpha } 1 \text { chain }\end{array}$ \\
\hline 12 & COL1A2 & $\begin{array}{l}\text { collagen type I } \\
\text { alpha } 2 \text { chain }\end{array}$ \\
\hline 13 & COL3A1 & $\begin{array}{l}\text { collagen type III } \\
\text { alpha } 1 \text { chain }\end{array}$ \\
\hline 30 & COL6A3 & $\begin{array}{l}\text { collagen type VI } \\
\text { alpha } 3 \text { chain }\end{array}$ \\
\hline 46 & COL14A1 & $\begin{array}{l}\text { collagen type XIV } \\
\text { alpha } 1 \text { chain }\end{array}$ \\
\hline 49 & COL6A1 & $\begin{array}{l}\text { collagen type VI } \\
\text { alpha } 1 \text { chain }\end{array}$ \\
\hline 66 & COL5A1 & $\begin{array}{l}\text { collagen type } \mathrm{V} \\
\text { alpha } 1 \text { chain }\end{array}$ \\
\hline 80 & COL8A2 & $\begin{array}{l}\text { collagen type VIII } \\
\text { alpha } 2 \text { chain }\end{array}$ \\
\hline 91 & COL6A2 & $\begin{array}{l}\text { collagen type VI } \\
\text { alpha } 2 \text { chain }\end{array}$ \\
\hline 118 & COL5A2 & $\begin{array}{l}\text { collagen type } \mathrm{V} \\
\text { alpha } 2 \text { chain }\end{array}$ \\
\hline 166 & COL10A1 & $\begin{array}{l}\text { collagen type } X \\
\text { alpha } 1 \text { chain }\end{array}$ \\
\hline
\end{tabular}
utilized in this study with respect to differential expression between tumor and metastasis tissues. This was also statistically significant (Table $1 ; p=5.42 E-08)$. 
COL1A2, collagen type I alpha 2 chain, was ranked 12th out of 22277 total transcripts measured and detected by the microarray dataset utilized in this study with respect to differential expression between tumor and metastasis tissues. This was also statistically significant (Table 1; $\mathrm{p}=2.88 \mathrm{E}-07$ ).

COL3A1, collagen type III alpha 1 chain, was ranked 13th out of 22277 total transcripts measured and detected by the microarray dataset utilized in this study with respect to differential expression between tumor and metastasis tissues. This was also statistically significant (Table $1 ; \mathrm{p}=6.02 \mathrm{E}-07$ ).

COL6A3, collagen type VI alpha 3 chain, was ranked 30th out of 22277 total transcripts measured and detected by the microarray dataset utilized in this study with respect to differential expression between tumor and metastasis tissues. This was also statistically significant (Table 1; $\mathrm{p}=7.62 \mathrm{E}-06)$.

COL14A1, collagen type XIV alpha 1 chain, was ranked 46th out of 22277 total transcripts measured and detected by the microarray dataset utilized in this study with respect to differential expression between tumor and metastasis tissues. This was also statistically significant (Table 1; $\mathrm{p}=2.19 \mathrm{E}-05)$.

COL6A1, collagen type VI alpha 1 chain, was ranked 49th out of 22277 total transcripts measured and detected by the microarray dataset utilized in this study with respect to differential expression between tumor and metastasis tissues. This was also statistically significant (Table 1; $\mathrm{p}=2.97 \mathrm{E}-05)$.

COL5A1, collagen type $V$ alpha 1 chain, was ranked 66th out of 22277 total transcripts measured and detected by the microarray dataset utilized in this study with respect to differential expression between tumor and metastasis tissues. This was also statistically significant (Table 1; $\mathrm{p}=8.57 \mathrm{E}-05)$.

COL8A2, collagen type VIII alpha 2 chain, was ranked 80 th out of 22277 total transcripts measured and detected by the microarray dataset utilized in this study with respect 
to differential expression between tumor and metastasis tissues. This was also statistically significant (Table 1; $p=1.15 E-04)$.

COL6A2, collagen type VI alpha 2 chain, was ranked 91st out of 22277 total transcripts measured and detected by the microarray dataset utilized in this study with respect to differential expression between tumor and metastasis tissues. This was also statistically significant (Table 1; $p=1.66 E-04)$.

COL5A2, collagen type $V$ alpha 2 chain, was ranked 118th out of 22277 total transcripts measured and detected by the microarray dataset utilized in this study with respect to differential expression between tumor and metastasis tissues. This was also statistically significant (Table 1; $\mathrm{p}=3.14 \mathrm{E}-04)$.

COL10A1, collagen type $X$ alpha 1 chain, was ranked 166th out of 22277 total transcripts measured and detected by the microarray dataset utilized in this study with respect to differential expression between tumor and metastasis tissues. This was also statistically significant (Table 1; $\mathrm{p}=6.98 \mathrm{E}-04)$.

Next, we extracted the exact mRNA expression levels of each collagen gene from each patient's tumor and metastasis samples to deteremine if the difference in expression between metastasis and tumor groups was statistically significant.

All genes were expressed at significantly lower levels in brain metastases than in primary tumors of the breast; these are matched samples (from the same patient). For COL1A1 (Figure 1A), p<0.0001; for COL15A1 (Figure 1A), $p<0.0001$; for COL1A2 (Figure 1A), $p<0.0001$; for COL3A1 (Figure 1A), $p=0.0001$; for COL6A3 (Figure 1B), $p<0.0001$; for COL14A1 (Figure 1B), $p<0.0001$; for COL6A1 (Figure 1B), $p<0.0001$; for COL5A1 (Figure 1B), $p<0.0001$; for COL8A2 (Figure 1C), $p=0.0002$; for COL6A2 (Figure 1C), $p=0.0002$; for COL5A2 (Figure 1C), $p=0.0011$; for COL10A1 (Figure 1C), $p=0.0014$. Thus, the decreased expression of all twelve collagen genes in each of the 16 brain metastasis samples compared to the primary breast tumors from which they arose was statistically significant. 
Finally, we asked whether the products of these genes had been shown experimentally to be interaction partners, in order to determine if these collagen gens might be part of a collagen interaction network. In fact, we found using STRING computational protein-protein interaction analysis (8) that COL5A1 interacted with six other collagens that were found to be differentially expressed in brain metastases here: COL6A1, COL6A2, COL6A3, COL1A1, COL1A2, and COL8A2 (Figure 2). A COL1A1 and COL1A2 interaction was also detected (Figure 2). Thus, 12 collagens are down-regulated at the level of mRNA in the brain metastases of patients with breast cancer, and 7 of these collagens are part of a protein interaction network with COL5A1 as its center.

\section{Discussion}

Breast cancer is a leading cause of death for women (1), and metastasis is the major cause of death in breast cancer (2). Breast cancer can metastasize to the brain, but the basic mechanisms that drive metastasis to the brain in breast cancer are still largely unknown (9). Novel therapies are required to prevent, delay, or treat metastases in breast cancer (3). To understand the basic transcriptional of brain metastases as they arise from primary tumors of the breast, we compared the transcriptomes of 16 primary breast tumors isolated from human patients to that of 16 matched brain metastases using a published dataset (4). Using the power of systems-level transcriptome analysis, we found that 12 of the top $1 \%$ of the most differentially expressed genes between the transcriptomes of brain metastases and primary tumors of the breast were genes of the collagen family. Computational protein-protein interaction analysis showed that 7 of these genes formed a protein-interaction network with COL5A1 as its center.

Collagens are structural components of the extracellular matrix (10), the architecture that surrounds each cell of solid tissues (11) and from which, during the theoretical migration and progression of tumor cell to circulating tumor cell to metastasis $(12,13)$, the tumor cell must dissolve, extract itself from, or otherwise perturb in order to colonize a foreign site (14). For this reason, the extracellular matrix has been termed by one group as "a gatekeeper in 
transition from dormancy to growth" (15). Remodeling of the extracellular matrix has been found in cancers of the cervix, prostate, lung, and ovaries $(16,17,18,19)$. Perturbed expression of collagens has been previously reported in breast cancer (20) but this is the first report to document perturbed expression of collagens in metastases to the brain in breast cancer.

We found that 7 out of 12 of these genes formed a protein-interaction network with COL5A1 at its center. Mutations in COL5A1 cause Ehlers-Danlos Syndrome, a disease primarily affecting the skin, ligaments and joints which manifests as tissue fragility, joint hypermobility, and hyperelasticity of the skin (21). Importantly, haploinsufficiency in COL5A1 is sufficient, demonstrating that a decrease in COL5A1 expression is associated with a pathological process that is conducive at the biophysical level to increased overall tissue elasticity (22). One possibility that is the same sort of tissue elasticity that is caused by a decrease in COL5A1 expression in Ehlers-Danlos contributes to the metastatic process in breast cancer.

Brain metastases affect $10-15 \%$ of women with breast cancer (8). We found that brain metastases down-regulate the expression of a series of 12 collage genes during the progression from breast tumor to brain metastasis. The protein products of 7 these genes form an interaction network centered on COL5A1. The effect of ectopic over-expression of each of these collagen genes, individually and in tandem, and specifically of COL5A1 on tumor progression and metastasis to the brain should be evaluated using xenograft mouse models $(23,24)$. Modulation of collagen expression could represent a novel therapeutic strategy to prevent or delay metastasis to the brain in women with breast cancer. 


\section{References}

1. American Cancer Society. "Breast cancer facts \& figures 2017-2018." (2017).

2. Mehlen, Patrick, and Alain Puisieux. "Metastasis: a question of life or death." Nature Reviews Cancer 6.6 (2006): 449.

3. Rostami, R., Mittal, S., Rostami, P., Tavassoli, F. and Jabbari, B., 2016. Brain metastasis in breast cancer: a comprehensive literature review. Journal of neuro-oncology, 127(3), pp. 407-414.

4. Iwamoto, T., Niikura, N., Ogiya, R., Yasojima, H., Watanabe, K.I., Kanbayashi, C., Tsuneizumi, M., Matsui, A., Fujisawa, T., Iwasa, T. and Shien, T., 2019. Distinct gene expression profiles between primary breast cancers and brain metastases from pairmatched samples. Scientific reports, 9(1), pp.1-8.

5. Liang, Peng, and Arthur B. Pardee. "Analysing differential gene expression in cancer." Nature Reviews Cancer 3.11 (2003): 869.

6. Vuorio, E. and De Crombrugghe, B., 1990. The family of collagen genes. Annual review of biochemistry, 59(1), pp.837-872.

7. Gilkes, D.M., Semenza, G.L. and Wirtz, D., 2014. Hypoxia and the extracellular matrix: drivers of tumour metastasis. Nature Reviews Cancer, 14(6), pp.430-439.

8. De Paepe, A., Nuytinck, L., Hausser, I., Anton-Lamprecht, I. and Naeyaert, J.M., 1997. Mutations in the COL5A1 gene are causal in the Ehlers-Danlos syndromes I and II. American journal of human genetics, 60(3), p.547.

9. Weil, R.J., Palmieri, D.C., Bronder, J.L., Stark, A.M. and Steeg, P.S., 2005. Breast cancer metastasis to the central nervous system. The American journal of pathology, 167(4), pp. 913-920.

10. Kielty, C.M. and Grant, M.E., 2002. The collagen family: structure, assembly, and organization in the extracellular matrix. Connective tissue and its heritable disorders: molecular, genetic, and medical aspects, pp.159-221.

11. Frantz, C., Stewart, K.M. and Weaver, V.M., 2010. The extracellular matrix at a glance. Journal of cell science, 123(24), pp.4195-4200.

12. Nguyen, Don X., Paula D. Bos, and Joan Massagué. "Metastasis: from dissemination to organ-specific colonization." Nature Reviews Cancer 9.4 (2009): 274.

13. Plaks, Vicki, Charlotte D. Koopman, and Zena Werb. "Circulating tumor cells." Science 341.6151 (2013): 1186-1188. 
14. Cox, T.R. and Erler, J.T., 2011. Remodeling and homeostasis of the extracellular matrix: implications for fibrotic diseases and cancer. Disease models \& mechanisms, 4(2), pp. 165-178.

15. Barkan, D., Green, J.E. and Chambers, A.F., 2010. Extracellular matrix: a gatekeeper in the transition from dormancy to metastatic growth. European journal of cancer, 46(7), pp. 1181-1188.

16. Fullár, A., Dudás, J., Oláh, L., Hollósi, P., Papp, Z., Sobel, G., Karászi, K., Paku, S., Baghy, K. and Kovalszky, I., 2015. Remodeling of extracellular matrix by normal and tumorassociated fibroblasts promotes cervical cancer progression. BMC cancer, 15(1), pp.1-16.

17. Tuxhorn, J.A., Ayala, G.E., Smith, M.J., Smith, V.C., Dang, T.D. and Rowley, D.R., 2002. Reactive stroma in human prostate cancer: induction of myofibroblast phenotype and extracellular matrix remodeling. Clinical Cancer Research, 8(9), pp.2912-2923.

18. Gao, Y., Xiao, Q., Ma, H., Li, L., Liu, J., Feng, Y., Fang, Z., Wu, J., Han, X., Zhang, J. and Sun, Y., 2010. LKB1 inhibits lung cancer progression through lysyl oxidase and extracellular matrix remodeling. Proceedings of the National Academy of Sciences, 107(44), pp. 18892-18897.

19. Sherman-Baust, C.A., Weeraratna, A.T., Rangel, L.B., Pizer, E.S., Cho, K.R., Schwartz, D.R., Shock, T. and Morin, P.J., 2003. Remodeling of the extracellular matrix through overexpression of collagen VI contributes to cisplatin resistance in ovarian cancer cells. Cancer cell, 3(4), pp.377-386.

20. Kauppila, S., Stenbäck, F., Risteli, J., Jukkola, A. and Risteli, L., 1998. Aberrant type I and type III collagen gene expression in human breast cancer in vivo. The Journal of Pathology: A Journal of the Pathological Society of Great Britain and Ireland, 186(3), pp.262-268.

21. Steinmann, B., Royce, P.M. and Superti-Furga, A., 2002. The Ehlers-Danlos syndrome. Connective tissue and its heritable disorders, 2, pp.431-523.

22. Wenstrup, R.J., Florer, J.B., Willing, M.C., Giunta, C., Steinmann, B., Young, F., Susic, M. and Cole, W.G., 2000. COL5A1 haploinsufficiency is a common molecular mechanism underlying the classical form of EDS. The American Journal of Human Genetics, 66(6), pp. 1766-1776.

23. Liu, H., Kato, Y., Erzinger, S.A., Kiriakova, G.M., Qian, Y., Palmieri, D., Steeg, P.S. and Price, J.E., 2012. The role of MMP-1 in breast cancer growth and metastasis to the brain in a xenograft model. BMC cancer, 12(1), p.583. 
24. Baschnagel, A., Russo, A., Burgan, W.E., Carter, D., Beam, K., Palmieri, D., Steeg, P.S., Tofilon, P. and Camphausen, K., 2009. Vorinostat enhances the radiosensitivity of a breast cancer brain metastatic cell line grown in vitro and as intracranial xenografts. Molecular cancer therapeutics, 8(6), pp.1589-1595. 
Table 1-1

\begin{tabular}{|c|c|c|c|c|c|c|c|}
\hline Rank & ID & p-value & $\mathbf{t}$ & B & $\log F C$ & Gene & Gene name \\
\hline 2 & 217430_x_at & 1.02E-08 & -7.2980851 & 9.4654 & -3.0133 & COL1A1 & $\begin{array}{l}\text { collagen type I } \\
\text { alpha } 1 \text { chain }\end{array}$ \\
\hline 6 & 203477_at & 5.42E-08 & -6.7587495 & 7.9983 & -3.0264 & COL15A1 & $\begin{array}{l}\text { collagen type XV } \\
\text { alpha } 1 \text { chain }\end{array}$ \\
\hline 12 & 202403_s_at & 2.88E-07 & -6.2256148 & 6.5213 & -2.8297 & COL1A2 & $\begin{array}{l}\text { collagen type I } \\
\text { alpha } 2 \text { chain }\end{array}$ \\
\hline 13 & 211161_s_at & 6.02E-07 & -5.99094 & 5.8653 & -2.6813 & COL3A1 & $\begin{array}{l}\text { collagen type III } \\
\text { alpha } 1 \text { chain }\end{array}$ \\
\hline 30 & 201438_at & 7.62E-06 & -5.1830936 & 3.5983 & -3.5725 & COL6A3 & $\begin{array}{l}\text { collagen type VI } \\
\text { alpha } 3 \text { chain }\end{array}$ \\
\hline 46 & 216865_at & 2.19E-05 & -4.8447826 & 2.6545 & -2.5706 & COL14A1 & $\begin{array}{l}\text { collagen type XIV } \\
\text { alpha } 1 \text { chain }\end{array}$ \\
\hline 49 & 213428_s_at & 2.97E-05 & -4.7462592 & 2.3814 & -1.5638 & COL6A1 & $\begin{array}{l}\text { collagen type VI } \\
\text { alpha } 1 \text { chain }\end{array}$ \\
\hline 66 & 212488_at & 8.57E-05 & -4.400236 & 1.4314 & -1.6346 & COL5A1 & $\begin{array}{l}\text { collagen type } V \\
\text { alpha } 1 \text { chain }\end{array}$ \\
\hline 80 & 221900_at & 1.15E-04 & -4.3030477 & 1.1678 & -2.1471 & COL8A2 & $\begin{array}{l}\text { collagen type VIII } \\
\text { alpha } 2 \text { chain }\end{array}$ \\
\hline 91 & 213290_at & 1.66E-04 & -4.181881 & 0.8415 & -1.7693 & COL6A2 & $\begin{array}{l}\text { collagen type VI } \\
\text { alpha } 2 \text { chain }\end{array}$ \\
\hline 118 & 221730_at & $3.14 \mathrm{E}-04$ & -3.9670459 & 0.2703 & -2.4179 & COL5A2 & $\begin{array}{l}\text { collagen type } V \\
\text { alpha } 2 \text { chain }\end{array}$ \\
\hline 166 & 205941_s_at & 6.98E-04 & -3.6932647 & -0.4411 & -2.0705 & COL10A1 & $\begin{array}{l}\text { collagen type } X \\
\text { alpha } 1 \text { chain }\end{array}$ \\
\hline
\end{tabular}

Table 1: Collagen genes are among the most differentially expressed genes when comparing the transcriptomes of primary breast tumors and brain metastases from humans.

The rank of differential expression with respect to 22277 total transcripts, Affymetrix ID, pvalue, $t$, a moderated t statistic, $B$, the log-odds of differential expression between the two groups, log-fold change of gene expression, the gene symbol and gene name are shown in the table above. 

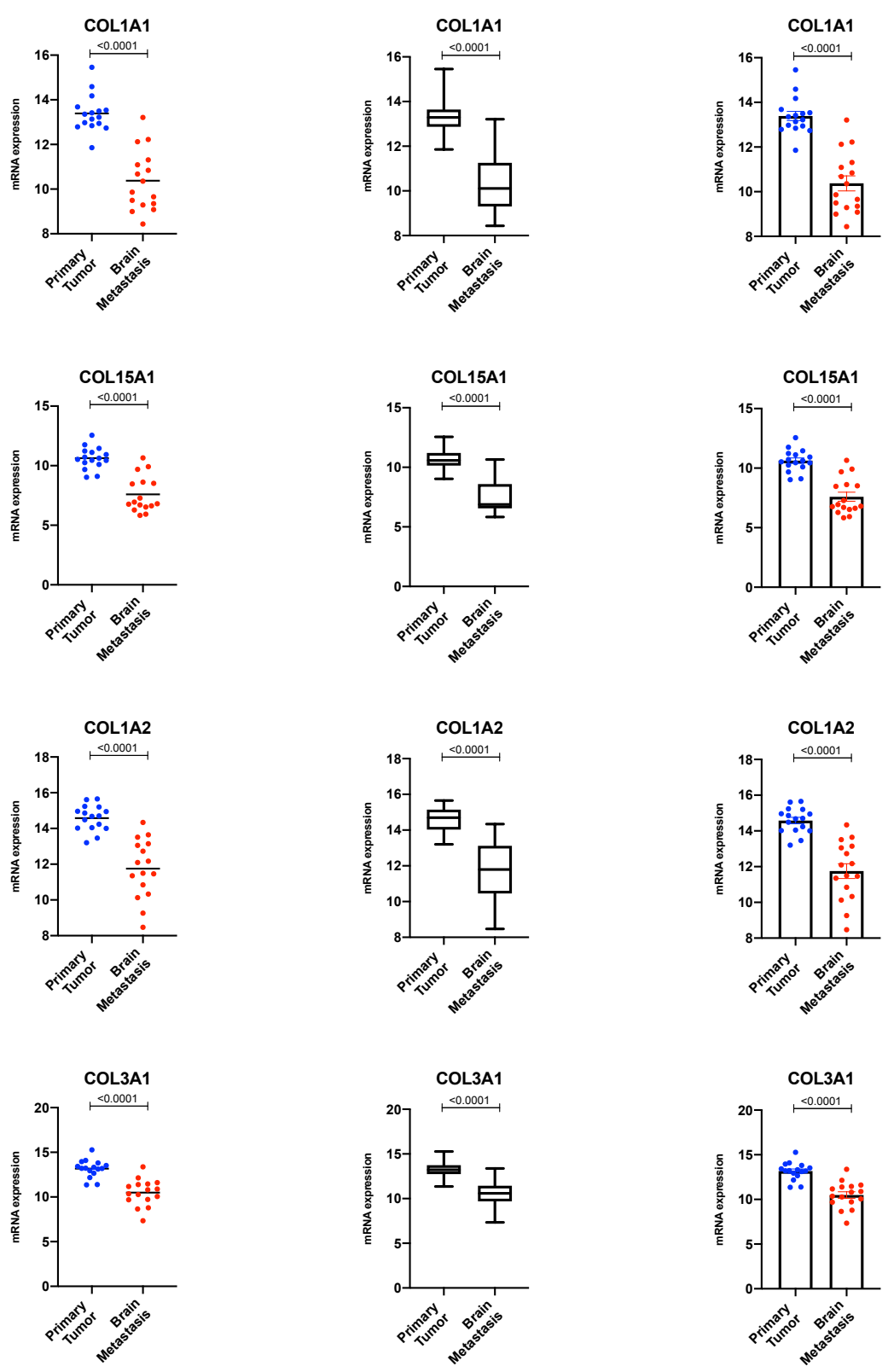
Figure 1A: The gene expression of multiple collagens is significantly lower in brain
metastases when compared to the primary breast tumors from which they arose.

The mRNA expression values of each collagen gene is graphically in blue (primary tumor) and red (brain metastasis), represented by dots denoting the expression level in each individual patient, with a black horizontal line denoting the mean in each group (left), by box and whisker plot from minimum to maximum value with a horizontal line denoting the median (middle), and by bar graph with the error bars denoting the standard error of the mean (right). The results of a statistical test evaluating the significance of difference between mean expression values between primary tumor and metastasis groups is shown as a p-value on each graph. 

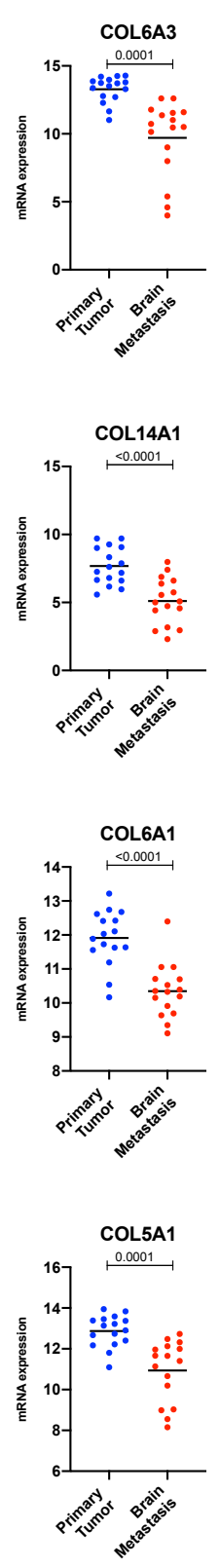
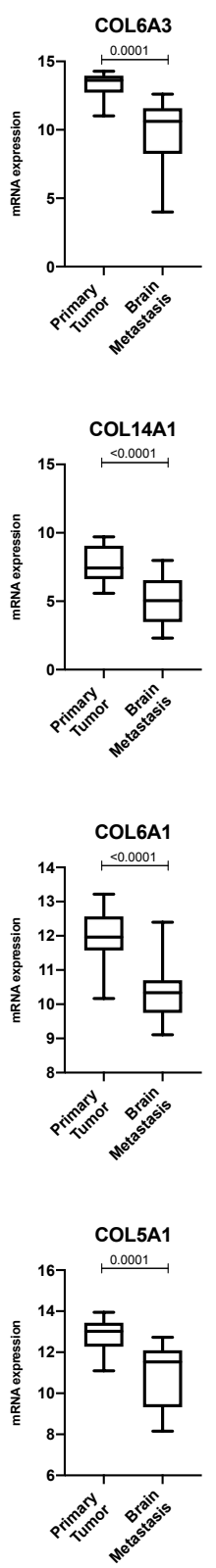
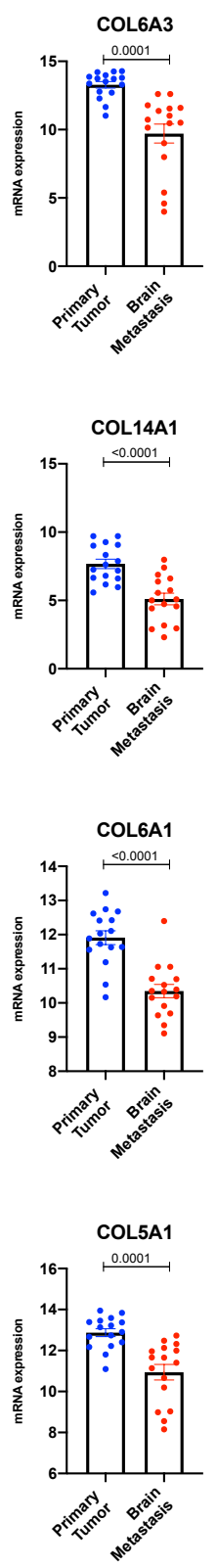

Figure 1B: The gene expression of multiple collagens is significantly lower in brain metastases when compared to the primary breast tumors from which they arose.

The mRNA expression values of each collagen gene is graphically in blue (primary tumor) and red (brain metastasis), represented by dots denoting the expression level in each individual patient, with a black horizontal line denoting the mean in each group (left), by box and whisker plot from minimum to maximum value with a horizontal line denoting the median (middle), and by bar graph with the error bars denoting the standard error of the mean (right). The results of a statistical test evaluating the significance of difference between mean expression values between primary tumor and metastasis groups is shown as a p-value on each graph. 

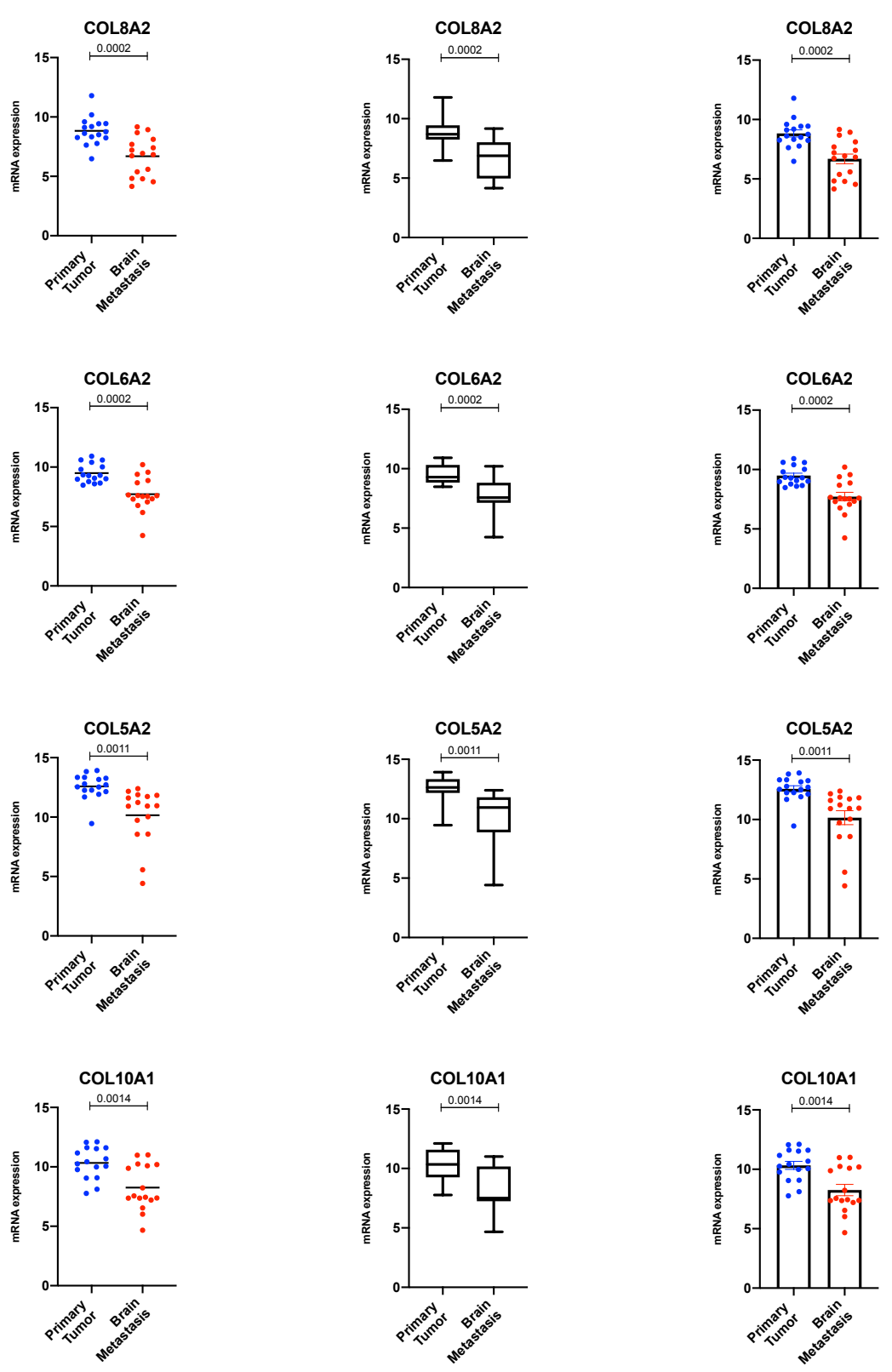
Figure 1C: The gene expression of multiple collagens is significantly lower in brain
metastases when compared to the primary breast tumors from which they arose.

The mRNA expression values of each collagen gene is graphically in blue (primary tumor) and red (brain metastasis), represented by dots denoting the expression level in each individual patient, with a black horizontal line denoting the mean in each group (left), by box and whisker plot from minimum to maximum value with a horizontal line denoting the median (middle), and by bar graph with the error bars denoting the standard error of the mean (right). The results of a statistical test evaluating the significance of difference between mean expression values between primary tumor and metastasis groups is shown as a p-value on each graph. 


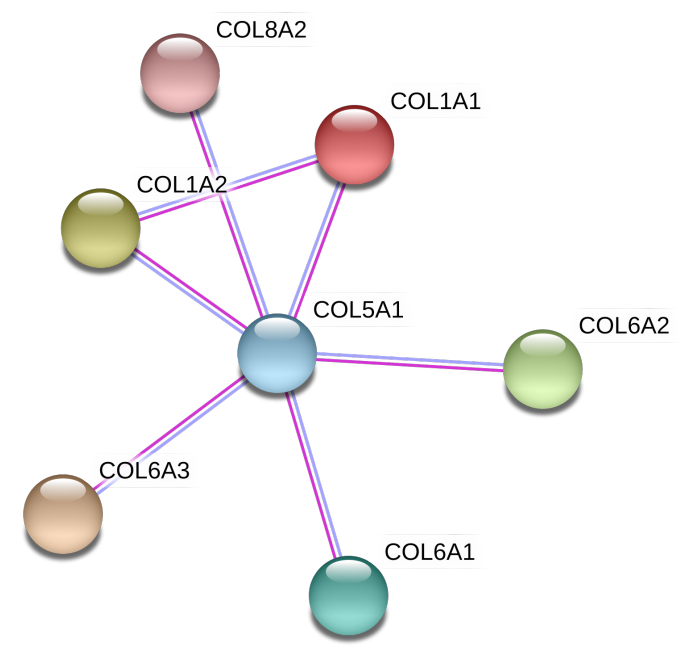

Figure 2: Protein-protein interaction analysis reveals a COL5A1 centered network involving COL6A1, COL6A2, COL6A3, COL1A1, COL1A2, and COL8A2.

All twelve differentially expressed collagen genes identified by transcriptome comparison of breast tumors and brain metastases were subject to STRING computational analysis of protein-protein network interaction. In this query, evidence was set to low confidence but the type of evidence that was allowed to be considered was purely experimental. 